\title{
Intra oral periapical radiography - basics yet intrigue: A review
}

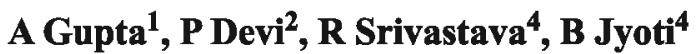

Abstract

There had been a long standing requirement for dental students which would help them to understand the basic techniques for intraoral periapical radiographs. Intra oral periapical radiography is an adjunct to the clinical examination and provides useful information about the joint components. The periapical view shows the entire crown and root of the teeth which provides vital information to aid in the diagnosis of the most common dental diseases. This article highlights the basic principle, techniques, advantages and disadvantages of intraoral periapical radiography.
\end{abstract}

Key words: Periapical radiographs, paralleling technique, bisecting technique.

\section{Introduction}

Radiographic examinations are one of the primary diagnostic tools used in dentistry to determine disease states and formulate appropriate treatment. ${ }^{1}$ Dr. Otto Walkhoff is credited with the first dental radiograph. Dental radiographs are valuable diagnostic tools when the image quality is adequate for proper interpretation ${ }^{2}$ Dental radiography is consistently under-utilized in veterinary practice. In many procedures, diagnostic radiographs are essential for the production of a treatment plan and treatment may be contraindicated without them. ${ }^{3}$ Periapical radiographs ("peri" meaning "around" and apical meaning "apex" or end of tooth root) record images of the outlines, position and mesiodistal extent of the teeth and surrounding tissues.

1. Dr. Anuj Gupta BDS, Intern, Department of Oral medicine and Radiology, Rama Dental College Hospital and Research Centre, Kanpur, Uttar Pradesh, India.

2. Dr. Parvathi Devi MDS, Professor, Department of Oral medicine and Radiology, Teerthankar Mahaveer Dental College Hospital and Research Centre, Moradabad, Uttar Pradesh, India.

3. Dr. Rahul Srivastava MDS, Senior Lecturer, Department of Oral medicine and Radiology, Rama Dental College Hospital and research Centre, Kanpur, Uttar Pradesh, India.

4. Dr. Bhuvan Jyoti MDS, Dental Surgeon and ConsultantOral Medicine and Radiology, Department of Dental Surgery, Ranchi Institute of Neuro-Psychiatry and Allied sciences, Ranchi, Jharkhand, India.

\section{Address of Correspondence:}

Dr. Anuj Gupta BDS, Intern, Department of Oral medicine and Radiology, Rama Dental College Hospital and Research Centre, 58/60 Neelwali gali, Birhana Road, Kanpur, Uttar Pradesh-208001., India.

E-mail : guptanuj712@gmail.com
In periapical radiograph it is essential to obtain the full length of the tooth and at least $2 \mathrm{~mm}$ of the periapical bone. ${ }^{4}$ The purpose of the intraoral periapical examination is to obtain a view of the entire tooth and its surrounding structures. ${ }^{5}$ Intra oral Periapical radiography is a commonly used intraoral imaging technique in dental radiology and may be a component of intraoral periapical radiologic examination. Periapical radiographs provide important information about the teeth and surrounding bone. The film shows the entire crown and root of the teeth and surrounding alveolar bone which provides vital information to aid in the diagnosis of the most common dental diseases; specifically tooth decay, tooth abscesses and periodontal bone loss or gum disease. Additional important findings may be detected, including the condition of restorations, impacted teeth or broken tooth fragments and variations in tooth and bone anatomy. 6

\section{Need of Intraoral Periapical Radiographs}

Periapical radiograph should show all of a tooth including surrounding bone. ${ }^{7}$ There are certain indications for the intra oral periapical radiography that includes:

\section{Assessment of Periodontal Status:-}

Intra oral periapical radiographs can be use for assessment of periodontal status for following significant features:

1. Receding bone height related to C.E.J.

2. Loss of bone at interproximal space or at furcation.

3. Widening of periodontal space.

4. Loss of integrity of lamina Dura. 


\section{Exodontia:-}

1. For diagnosis and planning of treatment of fractured teeth.

2. For the distinguistion between complicated crown fracture (pulpaly exposed) and uncomplicated crown fracture (not pulpaly exposed).

3. Pre extraction planning for the developmental anomalies. 4. Assessment of root morphology, resorptive lesion and ankylosis.

5. Post extraction radiographs for root fragments and other co-lateral damages.

\section{Conservative/Operative Procedures:-}

1. Detection of the dental caries.

2.Intraoperative or post operative radiographs for the demonstration of file, position of gutta percha point in canal, and adequate filling of pulp canal.

3. Internal resorption, detection of developmental anomalies.

4. Detection of missing teeth, teeth with developmental anomalies E.g.:- dilacerations, supernumerary teeth, fusion etc. 5. Evaluation of implant postoperatively. ${ }^{3,8}$

\section{Technique for Periapical Radiography:-}

Two exposure techniques are implemented for periapical radiography. Prior to presenting technique a clear of understanding of the technique must be established, although the bisecting angle technique is utilized by the practitioners, the paralleling technique is the method of choice for intraoral radiography. The paralleling angle technique provides less image distortion and reduces excess radiation to the patient. ${ }^{5,9}$

\section{The Paralleling Technique:-}

The paralleling technique is also known as "right angle technique" or "long cone technique". It is used for both periapical and bitewing radiographs. In the case of periapical radiograph the film receptor should be placed parallel to the crown and root of the teeth being imaged and the central ray of the $\mathrm{x}$-ray beam is directed at the right angle to the teeth and film. The orientation of the film, teeth and the central ray minimized the geometric distortion. ${ }^{1,7}$ (Fig.1)

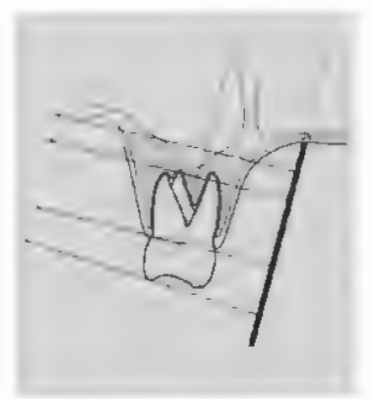

Fig 1: Paralleling Technique: diagram of paralleling technique illustrating the relationship of the film and the teth in the paralleling (right angle) technique.
The Paralleling principle, in essence, follows the five rules of accurate image formation to minimize the undesirable image characteristics of unsharpness, magnification and shape distortion. These rules are as follows:

1. The $x$-rays should originate from as small a focal spot as possible, that determined by manufacturer. Efforts should be made to minimize voluntary and involuntary motion unsharpness of film or $\mathbf{x}$-ray tube. This will increase the size of the focal spot.

2. The distance between the focal spot and the object to be examined should always be as long as is practical.

3. The film should be as close as possible to the object being radiographed.

4. As far as is practical, the long axis of the object should be parallel to the film.

5 . The central ray should be as nearly perpendicular to the film as possible to record the adjacent structures in their true spatial relationship. ${ }^{4}$

\section{Instruments:-}

The film holder consists of the 3 basic components:

1. A mechanism for holding the film packet parallel to the teeth that prevents the bending of the film.

2. A bite block or platform.

3. Beam Aiming Device:-This may or may not prevent the collimation of the beam. ${ }^{8}$

A number of commercial devices are available that will hold the film parallel and at varying distances from the teeth:

1. The XCP instruments (extension cone paralleling)

2. The precision rectangular collimating instruments which restrict the beam size at the patient's face to the size of radiograph.

3.The Stabe disposable film holder.

4. The snap- A-Ray intra oral film holder

5.A haemostat inserted through a flattened rubber bite bock which will serve in much the same manner as the Snap-A- Ray film holder. ${ }^{10}$

The choice of holder is a matter of personal preference, RIN XCP holders as shown in the Fig.2.

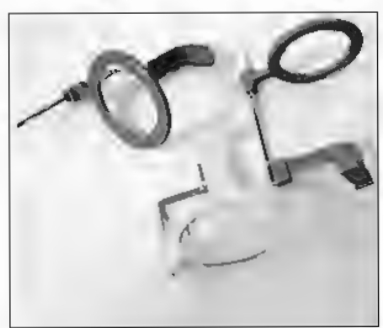

Fig 2: Yellow colour coded posterior holder (RNN XCP), Blue colour coded anterior holder (RIN XCP), Red colour coded superbite posterior holder (for bitewing). 


\section{Technique:-}

Select the appropriate holder and the film

Maxillary and Mandibular Incisors \& Canine

The anterior holder should be used and the small film packet with its long axis vertical,

Maxillary and Mandibular Premolar \& Molar

Posterior holder should be used (right or left) as per the case and large film packet (31x 41) $\mathrm{mm}$ with its long axis horizontal.

\section{Key Points:-}

1. Smooth white surface of the film must face towards the $x$-rity sources.

2. End of the film packet with imposed orientation dot should place opposite to the crown of the tooth.

3. Patient is positioned with head support with occlusal plane horizontal.

Placement of The Film In Patlent's Mouth:-

For Maxillary Incisors \& Canine:-

Placed the film sufficiently posterior to enabled it's hight to be accommodate in palatal veult.

For Mardlary Premolar \& Malar:-

Place the film in the middle of the palate to accommodate its height in palatal vault.

\section{For Mandibular Incisor \& Canine:-}

Film is positioned in lime with the lower canine or first premolar.

For Mandibular Premolar \& Molars:-

Film is placed in lingual sulcus next to appropriate teeth.

The holder is rotated so that teeth to be imaged a touching the bite block placed the cotton wool roll on the reverse side of the bite block to keep the tooth $k$ film parallel and make holder comfortable to stabilize bolder. Ask the patient to bite gently. Locator ring is moved down the indicator rod until this just in contact with the patient face to obtrin the comect focal spot to the film distance. Although the beam indicating device with locator ring this automatically both vertical and horizontal angles and centres the beam over the film. Exposure is made. ${ }^{8}$

Point of entry for different teeth used in paralleling technique is described in Table. $1^{10}$

Table 1: Point of entry for different teeth ased in paralleling technique

\begin{tabular}{|c|c|}
\hline IMAGE FIID & FinT OF ENTHY OF GRTIRAL GAY \\
\hline 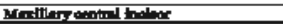 & 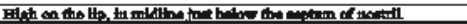 \\
\hline 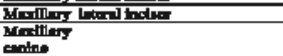 & 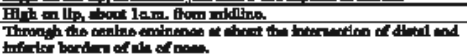 \\
\hline Promoly & 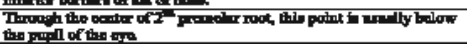 \\
\hline Wring & 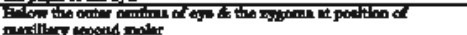 \\
\hline 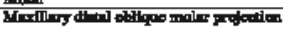 & 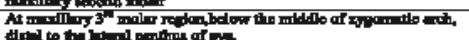 \\
\hline 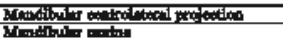 & 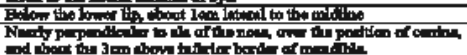 \\
\hline 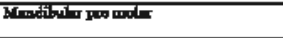 & 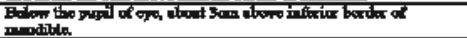 \\
\hline 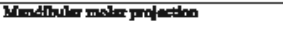 & 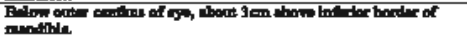 \\
\hline 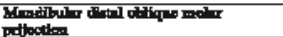 & 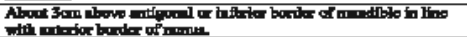 \\
\hline
\end{tabular}

\section{Advantages:-}

1. Less magnification so geometrically accurate image can be produced.

2. The shadow of zygomatic process appears above apices of the molar teeth.

3. Good representation of periodontal bone level.

4. Minimal foreshortening of elongation gives accurate infurmation about the periapical repion of image of teeth.

5. Detection of a proximal caries because of well demonstration of crown of the teeth.

6. Automatically determine vertical and horizontal angulations by positioning device if placed correctly.

7. No coning of or cone cutting as the $x$-ray beam is aimed accurately ay the centre of the film.

8. Reproducible radiographs are possible relative postures of film teeth and $x$ ray been is always maintained in respective of patient's position 7,8

\section{Dlsadvantages:-}

1. Positioning of the film can be uncomfortable for the posterior teeth.

2. Inexperienced operstor can face difficulty in placing the holders in the mouth.

3. Cannot be performed satisfactory using short focal spot to skin distrance.

4. Difficulty in placing the holder in lower third molar regions.

5. Holders used to be auto clave or disposable.

6. Cannot done in cases of shallow flat palate.

7. Sometimes apical region of the teeth may appear near the edge of the film 7,8

\section{Bisecting Angle Technique}

Bisecting angle technique is used for periapical radiography it is a useful alternative technique when ideal receptor placement cannot be achieved due to patient's trauma or anatomical obstacles sucb as tori, shallow palate, and shallow floor of the mouth, short frenum, or narrow arch witth. This technique is more operator sensitive. If angle is not correctly bisected elongation or foreshortening will occur. ${ }^{2}$

\section{Princtple:-}

The bisecting angle technique is besed on geometric principle that states that two triangles are equal if they have two equal angles and a common side, it is called "Scieszynsk's Rule of Isometry". Isometry is defined as equality of measurement when the rule of Isometry is applied to dental radiography. (Fig.3)

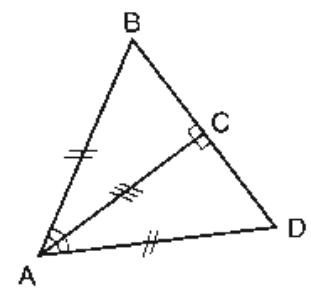

Fig 3: Rule of Igometry - Angle A is bisected by the line $\mathrm{AC}$. Lime $\mathrm{AC}$ is perpendicular to line BD. Angle DAC is equal to engle $\mathrm{CAB}$, and angle $\mathrm{ACD}$ in equal to ACB. If two triangles have to equal angles a common site, then it can be said that the two triangles are equal. Therefore, triangle DAC is equal to triangle $\mathrm{CAB}$. 
It is used to determine correct vertical angulations of cone (B.I.D.). Bisecting angle rule states that the central ray is directed through the medial plane of tooth, perpendicular to a line bisecting the angle formed by the plane of long axis of tooth and plane of the film. ${ }^{4,7}$ (Fig.4)

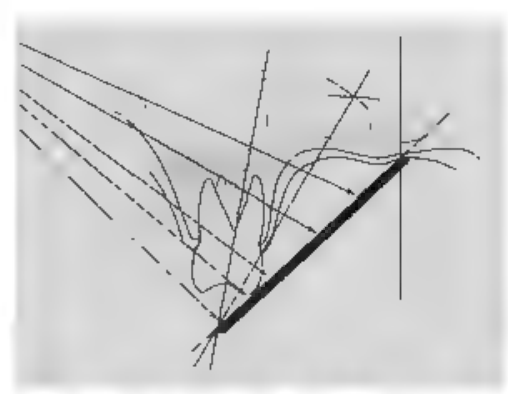

Fig 4: Bisecing Angle Technique: The rule of isometry apply to the intromal radiographie technique commonly ealled the bisection - of - the - angle technique. The path of central ray is directed perpendicular to a line binecting the angles fomed by the Iang yxis of the tooth and the plane of the film.

\section{Ingtruments Used:-}

Many methods can be used to support the file for bisecting angle technique. The preferred method is to use a film bolding instrument (SNAP-A-RAY) OR BISECTING TECHNIQUE INSTRUMENTS.

Two sucb film holders are the DENTSPLY/RINN BISECTING ANGLE INSTRUMENT (B.A.I.) film holders and the dentsply/rinn stable disposable periapical x-ray film holders.

\section{Dentuply/Rinn (B.A.I.) Inotrumentu:-}

These instruments are designed to add in the determination of horizontal and vertical angulations, minimized distortion film bending and preventing cone-cutting.

\section{Dentuplty /Rinn Stab Film Holder's:-}

These film holders are made of exposed /rigid poly styrene material that allowing the patient's teeth to penetrate the bite blocks portion and the film bolder, locking it into position.

\section{Technique:-}

When using the bisecting angle technique the head position is very important, the rule for head position or patient's positions as follows:-

1. The occlusal plane of teeth to be radiograpbed should be parallel to the floor.

2. Saggital plane should be perpendicular to the floor for maxillary Projection, here alatragus line is parallel to the floor.
3. In Mandibular projection, mandibular occlusal plane changes when mouth is opened, so patient's head should be tilted slightly backward so that occlusal plane of the mandible will parallel to the floor when mouth open. Here lip commissural -tragus line is parallel to the floor.

Place the film to set the tooth to be imaged as close as possible. The angle formed between long axis of tooth and long axís of film assessed and mentally bisected. Place the $\mathrm{x}$ ray tube at right angle of the bisecting line with central ruy aiming to the tooth apex. As per geometric principle of similar triangles, the length of tooth will be equal to length of the imaged tooth $4,7,8$

Point of entry for different teeth used in Bisecting Angle technique is described in Table $2^{10}$

Table 2: Point of entry for different teeth used in bisecting technique

\begin{tabular}{|c|c|}
\hline IMACE nIDID & 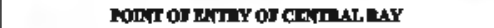 \\
\hline 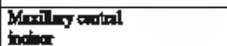 & 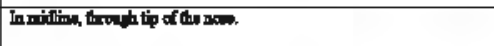 \\
\hline 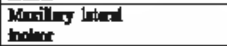 & 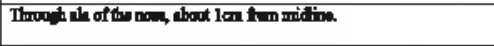 \\
\hline $\begin{array}{l}\text { Maning } \\
\text { enint }\end{array}$ & 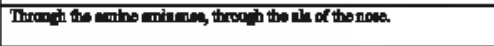 \\
\hline Mniling & 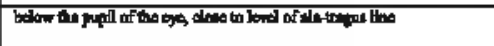 \\
\hline $\begin{array}{l}\text { Monity } \\
\text { Molr }\end{array}$ & 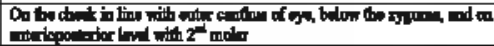 \\
\hline 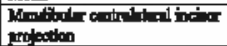 & 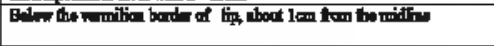 \\
\hline Mondhing anthe & 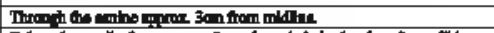 \\
\hline 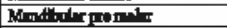 & 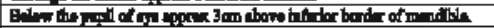 \\
\hline 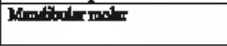 & 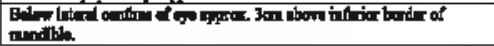 \\
\hline
\end{tabular}

\section{Vertical Angulation}

1. Rule for vertical angulations in bisecting angle techmique is to direct the central ray through the centre of the filed under examination, perpendicular to the line bisecting the angle formed by the planes of long axis of the tooth and the plane of the film.

2. Plus $(t)$ vertical angulations means cone is above the horizontal pointed downward.

3. Minus (-) vertical angulations means cone is below the horizontal pointed upward. The ranges of prescribed vertical angulations are listed in TABLE $34, f$

Table 3: Ranges of preacrlbed vertical unguintion: for bleecting angle technique

\begin{tabular}{|c|c|c|}
\hline PROJECTIONS & MAXILLA & MANDIBLE \\
\hline Incisors & $+40^{\circ}$ & $-15^{\circ}$ \\
\hline Canines & $+45^{\circ}$ & $-20^{\circ}$ \\
\hline Premolars & $+30^{\circ}$ & $-10^{\circ}$ \\
\hline Molars & $+20^{\circ}$ & $-5^{\circ}$ \\
\hline
\end{tabular}

\section{Horizontal Angulation}

1. In the horizontal plane the central ray should be aimed through the inter proximal contact areas.

2. Horizontal angulations deternined by shape of arch and position of tooth. 


\section{Periapical Radiography}

\section{Advantages:-}

1. Placement of the film is simple and quick.

2. Positioning of the file is comfortable in all areas of the mouth.

3. The image tooth will be the same length as tooth itself and should be adequate for diagnostic purpose if all angulations are used correctly.

\section{Disadvantages:-}

1. Periodontal bone levels are shown poorly.

2. Non reproducible.

3. The shadow of zygomatic process overlies the root of maxillary molars.

4. Horizontal \& vertical angles has to be assessed for every patient.

5. The technique required experienced operator.

6. An incorrect horizontal angulation causes overlapping of crown and root.

7. Incorrect vertical angulations will cause foreshortening or elongation of image.

8. Buccal roots of maxillary premolar and molar get foreshortened

9. Crown of teeth are often distorted thus preventing detection of proximal caries.

10. If central ray is not aimed at the centre of the film coning off or cone cut may result. ${ }^{7,8}$

\section{Conclusion}

Diagnostic intra oral periapical radiography has evolved as an inseparable branch of dentistry. Intra oral periapical radiography is an important diagnostic aid and routinely used for investigating the periapical and periodontal diseases. Proper intra oral periapical technique not only helps to confirm the diagnosis but also aids treatment planning and management and baseline for assessing the outcome of each pulpal, periapical and periodontal pathologies.

\section{References}

1. Williamson GF. Keys to Successful Intraoral Radiography. Available from: h t t p : / / w w w. Dentsply learning. com /contrib./documents/AL_HND_VOJK-TJ6081.20109 2092 1359510.pdf

2.Williamson GF. Intraoral Radiography: Positioning and Radiation Protection. Available from: http://www.ineedce.com /courses /1455/PDF / IntraoralRadiography.pdf

3. Dental radiography. Available from: http://www.rvc.ac.uk / review/dentistry/Shared_Media/ pdfs/Radiography_print.pdf

4. Langland OE, Langlais RP. Principles of Dental Imaging, 2nd Ed., Philadelphia: Lippincott Williams \& Wilkins, 2002;85-138.

5. Farman AG, Kolsom SA. Intraoral Radiographic Techniques. Available from: http://www.dentalcare.com/en-US/dental-education/con tinuing-education / ce119 / ce119 .aspx ? review=true.

6. Cash M, Perschbacher S. Periapical Radiographic Technique - Long Cone Parallelling - Patient. Available from: http: //www . dentistry . utoronto.ca /dpes/radiology/patients/periapical-radiographic-techni que-long-cone-parallelling-patient.

7. White SC, Pharoah MJ. Oral Radiology: Principles and Interpretation, 5th Ed. St. Louis: Mosby Inc., 2004.

8. Whaites E. Essentials of dental radiography and radiology. In: Periapical radiography. 3rd Ed. London,Churchill Livingstone 2002;75-100.

9. Williamson GF. Intraoral Radiography: Principles, Techniques and Error Correction. Available from: http://www. Dentalcare .com/en-US /dental-education/continuing-education /ce137 / ce 137.aspx?review=true.

10. Goaz PW, White SC. Oral Radiology: Principles and Interpretation. In: intra oral radiographic examinations. 3rd Ed., C.V. Mosby, 1994. 